\title{
Reversion of BDNF, Akt and CREB in Hippocampus of Chronic Unpredictable Stress Induced Rats: Effects of Phytochemical, Bacopa Monnieri
}

\author{
Somoday Hazra', Sourav Kumar², Goutam Kumar Saha ${ }^{1}$, and Amal Chandra Mondal ${ }^{2,3} \bowtie$ \\ ${ }^{1}$ Department of Zoology, University of Calcutta, Kolkata, West Bengal, India \\ ${ }^{2}$ Department of Physiology, Raja Peary Mohan College, Uttarpara, Hooghly, West Bengal, India \\ ${ }^{3}$ School of Life Sciences, Jawaharlal Nehru University, New Delhi, India
}

Objective The aims of the present study were to explore the behavioural effects and to understand the possible mode of action of Bacopa monnieri extract (BME) on chronic unpredictable stress (CUS) induced depressive model and the biochemical alterations such as brain derived neurotrophic factor (BDNF), Akt, cyclic-AMP response element binding (CREB) protein level in the hippocampus of rats.

Methods We examined the effects of chronic administration of BME on CUS exposed rats for 28 days. Behavioural changes were assessed by sucrose consumption and open field test to assess the effect of BME on CUS-induced depression. The mechanisms underlying antidepressant like action of BME was further evaluated by measuring levels of BDNF, Akt, and CREB in the hippocampus of rat brain and compared with the standard tricyclic antidepressant drug imipramine ( $20 \mathrm{mg} / \mathrm{kg}$ body weight).

Results Exposure to CUS for 28 days produced depression-like behavior in rats, as indicated by significant decreases in sucrose consumption, locomotor activity including decreased BDNF, Akt and CREB levels in the hippocampus. Daily administration of BME at a dose of ( $80 \mathrm{mg} / \mathrm{kg}$ body weight) significantly reverses the behavioral alteration and restored the normal level of BDNF, total and phospho-Akt, total and phospho CREB in the hippocampus of CUS induced rats as compared to vehicle treated control rats.

Conclusion These findings suggest that BME ameliorates CUS induced behavioural depression in rats and that can be used as a potent therapeutic agent in treating depressive like behavior.

Psychiatry Investig 2017;14(1):74-80

Key Words Akt, Antidepressant activity, Bacopa monnieri, BDNF, CREB, CUS, Hippocampus.

\section{INTRODUCTION}

Major depressive disorder (MDD) is a leading cause of disability worldwide. The clinical presentation of MDD consists of a spectrum of neuropsychiatric symptoms, including anxiety. ${ }^{1}$ Depression is now potentially life threatening disease which affects $17 \%$ population of the world. ${ }^{2}$ According to the study of World Health Organization, nearly 900000 people suicide every year worldwide. Depressive symptoms are characterized by low mood, anhedonia, irritability, insomnia or hy-

Received: August 17, 2015 Revised: December 25, 2015 Accepted: February 12, 2016 Available online: July 11, 2016

$\triangle$ Correspondence: Amal Chandra Mondal, $\mathrm{PhD}$

School of Life Sciences, Jawaharlal Nehru University, New Delhi 110067, India Tel: +91-11-2670-4505, Fax: +91-11-2674-2558

E-mail: amal_mondal@rediffmail.com

(a) This is an Open Access article distributed under the terms of the Creative Commons Attribution Non-Commercial License (http://creativecommons.org/licenses/bync/3.0) which permits unrestricted non-commercial use, distribution, and reproduction in any medium, provided the original work is properly cited. persomnia, psychomotor retardation or agitation, weight loss and suicidal tendencies. ${ }^{3}$ Monoamine oxidase inhibitors (MAOIs), tri-cyclic antidepressants (TCAs) such as selective serotonin re-uptake inhibitors (SSRIs), serotonin- norepinephrine re-uptake inhibitors (SNRIs) and norepinephrine re-uptake inhibitors (NRIs) are antidepressant drugs (AD) and clinically used for treatment of psychiatric disorders. These drugs target to the monoamine neurotransmitter systems to induce synaptic transmission and play an essential role in mood-related disorder. However, these drugs are associated with delayed onset of clinical efficacy and most of these drugs inconsistently effective and have unwanted side effects. ${ }^{4}$

Previous studies from our laboratory and others have shown various neuropharmacological effects of Bacopa monnieri extract (BME) ${ }^{3,5,6}$ BME may be an effective alternative for the treatment of neuropsychiatric diseases such as depression, anxiety disorders, clinical mental disorders, obsessive compulsive disorders, hysteria, epilepsy and insomnia. ${ }^{7}$ Bacosides A 
and $\mathrm{B}$, present in $\mathrm{BME}$ are the active principle constituents responsible for Bacopa's cognitive effects. ${ }^{8}$ Bacoside A and bacopasides, the chief constituents present in BME are responsible for its pharmacological effects. ${ }^{9}$ BME has also shown for its therapeutic effect in other stress induced models like tail suspension test, forced swim test and learned helplessness. ${ }^{3,10}$

Chronic unpredictable stress (CUS) in rodents is an established and most reliable model of depression. ${ }^{4}$ It leads to neurochemical and behavioral alterations that are analogous to those observed in depressed human patients, including increases in stress hormones, hippocampal atrophy, increased anxiety and depressive behaviors, and cognitive impairments. ${ }^{11}$ In CUS model, rats are exposed to different types of unpredictable stressors. Several types of behavioral tests including sucrose preference test, open field test ${ }^{5,12}$ and elevated plus maze were performed to assess anhedonia, depression and anxiety like behavior. $^{6}$

Neurotrophins the key molecules in specific brain regions is responsible for neuronal growth, maintenance, cellular differentiation and survival of neurons in the central nervous system. ${ }^{3}$ Neurotrophins include brain derived neurotrophic factor (BDNF), nerve growth factor (NGF), neurotrophins 3 (NT-3) and 4 (NT-4). BDNF is the best characterized among all neurotrophins because of its role in synaptic plasticity ${ }^{13}$ and its potential role in the disease pathology or treatment of many psychiatric diseases. ${ }^{14}$ Recent data suggest that mature BDNF activates different intracellular signaling pathways ${ }^{15}$ through its high-affinity tropomyosin receptor kinase $\mathrm{B}(\operatorname{TrkB})$ receptor. When BDNF is bound to cognate receptor TrkB, it induces its dimerization and the receptor tyrosine kinase is autophosphorylated, leading to activation of hippocampal intracellular signaling cascades. ${ }^{3}$ There are several signal transduction pathways found that BDNF-TrkB activation can regulate downstream molecules. ${ }^{16}$ The phosphatidylinositol 3-kinase (PI3K) pathway activates serine/threonine kinase and the mitogenactivated protein kinase (MAPK) or extracellular signal regulated kinase (ERK) pathway activate several downstream effectors. Each of these signaling pathways confers the unique function of BDNF on cells. ${ }^{17}$ Our recent findings show that depression like changes were reversed by chronic BME treatment. ${ }^{3,5,6}$ This present preclinical animal model suggests considerable protection against depression related neurodegeneration and to find out if it modulates downstream signalling pathways. Considering the information mentioned above, the aims of this present study was to investigate the $\mathrm{AD}$ like effect of BME on the hippocampal BDNF and its downstream signaling molecule like Akt and CREB protein in stress induced behavioral model of depression.

\section{METHODS}

\section{Animals}

Male Sprague-Dawley rats (180-220 gm) were obtained from the animal care and maintenance division of Chittaranjan National Cancer Institute (CNCI), Kolkata, India. The animals were maintained on a $12 \mathrm{~h}$ light/dark cycle (lights on at 6:00 a.m., lights off at 6:00 p.m.) under controlled temperature conditions $\left(22 \pm 2^{\circ} \mathrm{C}\right)$, and given standard food and water ad libitum. They were allowed to acclimatize for one week before experiments begin. The rats were randomly assigned to four groups, Vehicle control, CUS plus vehicle, CUS plus BME (80 $\mathrm{mg} / \mathrm{kg}$ b.w.) and CUS plus IMI (20 mg/kg b.w.). There were no significant changes found between normal control and vehicle control group rats after 4 week of experimental procedure (data not shown). All experiments conformed to the guidelines of the Committee for the Purpose of Control and Supervision of Experiments on Animal (CPCSEA, New Delhi). The experimental protocols were approved by the Institutional Animal Ethics Committee (IAEC) of Raja Peary Mohan College, Uttarpara, Hooghly (W.B.), India. Effort was made to minimize the number and suffering of the animals.

\section{Drugs}

BME was purchased ( $\geq 40 \%$ total bacosides) from Natural Remedies Pvt. Ltd., Bangalore, India and was prepared according to our previous protocol. ${ }^{3}$ Chemical structure and HPLC data of Bacoside A were shown in Figure 1. BME was administered intragastrically $60 \mathrm{~min}$ before each stressor applied at the dose of $80 \mathrm{mg} / \mathrm{kg}$ body weight (b.w.) once daily. ${ }^{3,6}$ Imipramine hydrochloride ( $\geq 99 \%$, crystalline) (IMI), a tricyclic antidepressant, was purchased from Sigma-Aldrich (St. Louis, MO, USA) and used as positive control for antidepressant action. IMI was applied intragastrically $60 \mathrm{~min}$ before each stressor applied at the dose of $20 \mathrm{mg} / \mathrm{kg}$ b.w. once daily. BME and IMI were dissolved in normal physiological saline $(0.9 \% \mathrm{NaCl})$ and the animals in the control and CUS groups were treated with equal volume of physiological saline as vehicle. ${ }^{6}$

\section{CUS procedures}

CUS procedure was performed with some modifications as described in our previous study. Unpredictable stressors like (1 $\mathrm{mA}, 15 \mathrm{~s}$ duration, average 1 foot shock/min) for $60 \mathrm{~min}, 5 \mathrm{~min}$ cold water swim (at $4^{\circ} \mathrm{C}$ ), $1 \mathrm{~min}$ tail pinch, $60 \mathrm{~min}$ restrain, $48 \mathrm{~h}$ food deprivation, $24 \mathrm{~h}$ water deprivation, and overnight illumination) was applied in random order to maximize the unpredictable nature of the stressors. The CUS procedure was carried out once per day for 28 days. ${ }^{6}$ 


\section{Sucrose preference test}

First, the rats were trained to adapt to two bottles of sucrose solution $(1 \%, \mathrm{w} / \mathrm{v})$. After that rats were deprived of water and food for $24 \mathrm{~h}$. The rats were given free access to two bottles containing $100 \mathrm{~mL}$ of sucrose solution $(1 \%, \mathrm{w} / \mathrm{v})$ and $100 \mathrm{~mL}$ of water respectively. After $60 \mathrm{~min}$, the volume of both the consumed sucrose solution and water was measured and recorded. ${ }^{6}$

\section{Open field test}

The open-field test was performed as described previously. ${ }^{6}$ In brief, apparatus consist of a square wooden arena (100 $\times$ $100 \times 50 \mathrm{~cm}$ ) with a black surface covering the inside walls. The floor of the wooden arena was divided equally into 25 squares marked by black lines. The room was kept in a dim light with a video camera placed centrally above the box for recording the events. Each animal was placed in the center of the box and allowed to explore freely for $5 \mathrm{~min}$. The number of crossings and rearing were recorded during the test period. This wooden apparatus was cleaned after every trial.

\section{Sample collection}

The rats were sacrificed by decapitation after twenty four hours of the last behavioral test. The whole brain of each rat was rapidly removed and chilled in an ice cold saline solution. Hippocampus were dissected on a cold plate and immediately frozen in liquid nitrogen. The tissue samples were stored at $-80^{\circ} \mathrm{C}$ until assay.

\section{Evaluation of BDNF, total \& phospho-Akt, total \& phospho CREB protein levels in the Hippocampus by Western Blot}

Western blot analysis was performed for BDNF, total and phospho -Akt, total and phospho CREB protein expression as described before. ${ }^{3}$ Tissues were homogenized in ice-cold lysis buffer (50 mM Tris, pH-8.0 $150 \mathrm{mM} \mathrm{NaCl}, 1 \% \mathrm{NP}-40,5 \mathrm{mM}$ EDTA, pH-8.0, 1\% deoxycholic acid) containing protease cocktail inhibitor (Sigma, St. Louis, MO, USA). Respective protein samples were electrophoresed on 15\% (W/V) SDS-polyacrylamide gel (Mini-PROTEAN ${ }^{\circledR}$ tetra cell with mini-trans Blot $^{\mathbb{B}}$, Bio-Rad, USA) and subsequently transferred from gel onto a nitrocellulose membrane (Millipore, USA). The membrane was incubated with fresh blocking buffer $(10 \mathrm{mM}$ Tris- $\mathrm{HCl}, \mathrm{pH}-$ 8.0, $150 \mathrm{mM} \mathrm{NaCl}, 0.05 \%$ Tween- 20 containing $5 \%$ non fat dried milk) for $1 \mathrm{~h}$ at room temperature and then probed with primary anti-BDNF, anti-Akt (total \& phospho) and anti-CREB (total \& phospho) and the monoclonal $\beta$-actin (Santa Cruz Biotechnology, Inc., USA), at $4^{\circ} \mathrm{C}$ overnight. Nitrocellulose membranes were washed three times in TBST and incubated in the appropriate HRP conjugated secondary antibody at room temperature for $2 \mathrm{~h}$ and washed three times in the TBST buffer. Immunoreactive bands were visualized by using the ECL substrate solution (Santa Cruz Biotechnology, Inc., USA). The electrophoresis image analysis system (Smartview 2001, S/ $\mathrm{N}$ : SV-0002202, Japan) was used for analysis of the optical density (OD) value of each band and normalized against corresponding $\beta$-actin band.

\section{Statistical analysis}

The Statistical Package for the Social Science (SPSS) 15.0 was utilized for statistical analyses. All data were expressed as mean \pm SEM and have been statistically analyzed with one way ANOVA followed by Student's t-test. $\mathrm{p}<0.05$ were considered as statistically significant.

\section{RESULTS}

\section{Sucrose preference test}

One way ANOVA showed significant variation of sucrose consumption $\left(\mathrm{F}_{3,39}=8.23 ; \mathrm{p}<0.001\right)$ among all the rat groups due to exposure of four week of CUS along with treatment of BME shown in Figure 2. CUS induced animals consumed significantly less amount of sucrose $(\mathrm{p}<0.05)$ compared to the vehicle controls. Chronic BME treatment of $80 \mathrm{mg} / \mathrm{kg}$ significantly increases the sucrose consumption in CUS exposed rats $(\mathrm{p}<0.05)$ compared to vehicle treated rats. Administration of $20 \mathrm{mg} / \mathrm{kg}$ IMI significantly increased the level of sucrose consumption in CUS exposed rats $(\mathrm{p}<0.05)$. There was no significant difference in the percentage of sucrose consumption among all rats before CUS treatment (data not shown).

\section{Open field test}

One way ANOVA indicated significant variation in the locomotor activity i.e. the number of crossing $\left[\left(\mathrm{F}_{3,39}=5.69 ; \mathrm{p}<0.001\right)\right.$ and rearing $\left.\left(\mathrm{F}_{3,39}=6.32 ; \mathrm{p}<0.001\right)\right]$ in the open field test shown in Figure 3. Further, after exposure of CUS, number of crossing $(p<0.05)$ and rearing $(p<0.05)$ were significantly decreased in the CUS group rats compared to the vehicle control group rats. Chronic treatment with BME $(80 \mathrm{mg} / \mathrm{kg}$ b.w.) significantly increased the number of crossing $(\mathrm{p}<0.05)$ and rearing $(\mathrm{p}<0.05)$ in the open field than the CUS grouped rats. Treatment with IMI (20 mg/kg b.w.) also significantly increased the number of crossing $(\mathrm{p}<0.05)$ and rearing $(\mathrm{p}<0.05)$. There were no significant differences found in the number of crossing and rearing among all rats before CUS procedure (data not shown).

\section{Effect of BME on BDNF, total \& phospho-Akt, total \& phospho CREB protein levels in Hippocampus by Western Blot}

One-way ANOVA depicted that BDNF levels significantly differed in the hippocampus $\left(\mathrm{F}_{3,63}=28.09, \mathrm{p}<0.001\right)$ among all 


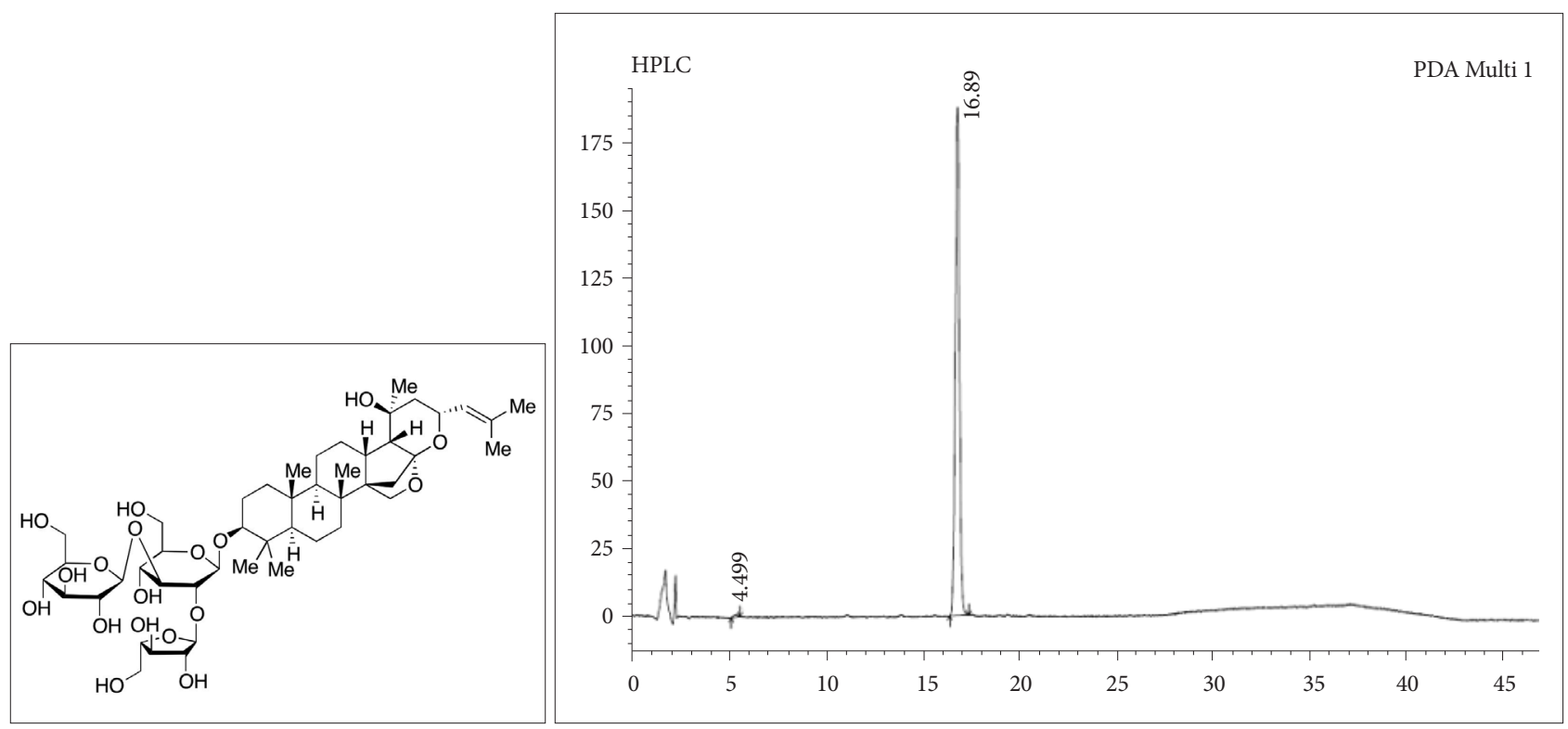

Figure 1. Chemical structure and HPLC data of Bacoside A.

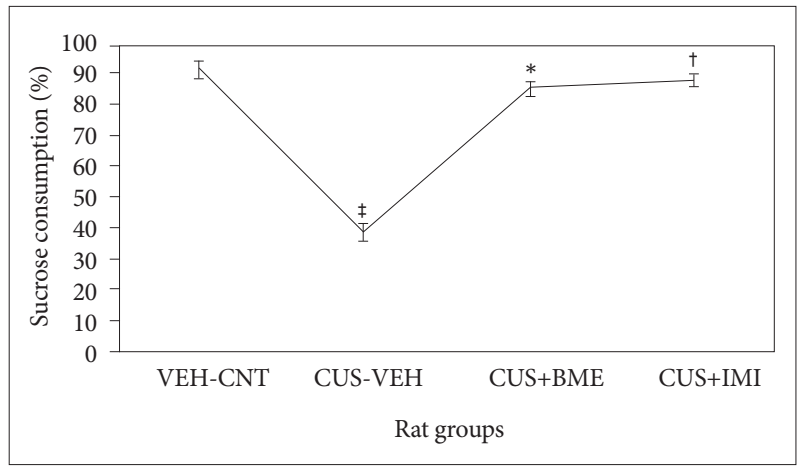

Figure 2. Effect of BME on percentage of sucrose consumption in CUS exposed rats. Values given are the mean \pm SEM $(\mathrm{N}=8)$. ${ }^{*} p<0.05,{ }^{\dagger} p<0.05$ as compared with the CUS group, ${ }^{\ddagger} p<0.05$, as compared with the Vehicle control group. VEH: vehicle, CNT: control, CUS: chronic unpredictable stress, BME: Bacopa monnieri extract, IMI: Imipramine.

the rat groups shown in Figure 4. BDNF levels in the hippocampus significantly reduced after CUS procedure in CUS group compared to vehicle control group $(\mathrm{p}<0.001)$. Chronic treatment with BME $(80 \mathrm{mg} / \mathrm{kg}$ b.w.) had significantly increased BDNF level in the hippocampus than CUS group rats $(p<0.001)$, suggesting that BME had a reversing effect on BDNF level in hippocampus. Administration of IMI $(20 \mathrm{mg} /$ $\mathrm{kg}$ b.w.) also showed similar effects like BME by increasing BDNF level significantly in hippocampus compared to CUS group rats $(\mathrm{p}<0.001)$.

One way ANOVA revealed that significant changes of total and phospho-Akt level among all rat groups in hippocampus $\left(\mathrm{F}_{3,63}=22.81, \mathrm{p}<0.001\right.$ and $\mathrm{F}_{3,63}=37.56, \mathrm{p}<0.001$ respectively $)$ shown in Figure 5. BDNF levels in the hippo-campus significantly reduced as compared to vehicle control group $(\mathrm{p}<0.001)$

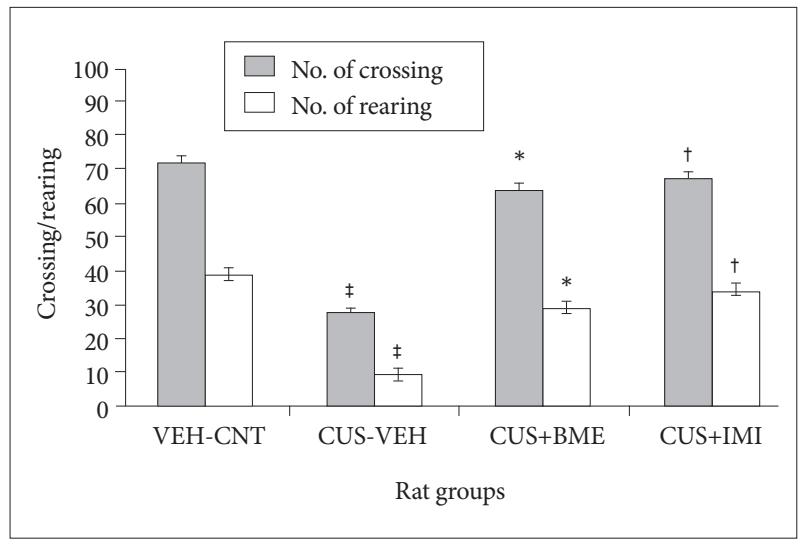

Figure 3. Effect of BME on crossing and rearing in the open field test in CUS exposed rats. Values given are the mean \pm SEM $(N=8)$. ${ }^{*} p<0.05,{ }^{\dagger} p<0.05$ as compared with the CUS group, $\neq p<0.05$ as compared with the vehicle control group. VEH: vehicle, CNT: control, CUS: chronic unpredictable stress, BME: Bacopa monnieri extract, IMI: Imipramine.

after exposure to CUS for 4 weeks. Significant increase of totalAkt $(\mathrm{p}<0.001)$ and phospho-Akt $(\mathrm{p}<0.001)$ were observed in BME treated rat group than CUS group rats, suggesting that BME had a reversing effect on total and phospho-Akt level in hippocampus. Treatment with IMI ( $20 \mathrm{mg} / \mathrm{kg}$ b.w. $)$ as positive control also showed similar effects like BME by increasing total-Akt $(\mathrm{p}<0.001)$ and phospho-Akt $(\mathrm{p}<0.001)$ significantly in hippocampus than CUS group rats. One-way ANOVA indicated that the total-CREB and phospho-CREB level significantly differed in hippocampus among rat groups $\left(\mathrm{F}_{3,63}=11.52\right.$, $\mathrm{p}<0.001)\left(\mathrm{F}_{3,63}=6.74, \mathrm{p}<0.001\right)$ respectively shown in Figure 6. After four weeks of CUS paradigm, total-CREB and phosphoCREB level in hippocampus significantly reduced in CUS 


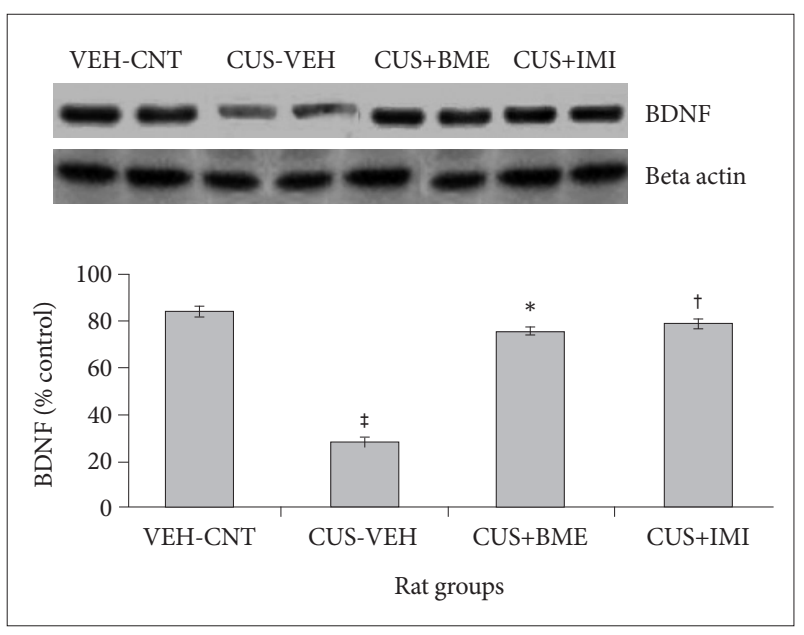

Figure 4. Effect of BME on BDNF protein level in the hippocampus of CUS exposed rats. Values given are the mean \pm SEM $(\mathrm{N}=8) .{ }^{*} \mathrm{p}<0.001,{ }^{\dagger} \mathrm{p}<0.001$ as compared with the CUS group, $\neq_{p}<0.05$ as compared with the vehicle control group. VEH: vehicle, CNT: control, CUS: chronic unpredictable stress, BME: Bacopa monnieri extract, IMI: Imipramine.

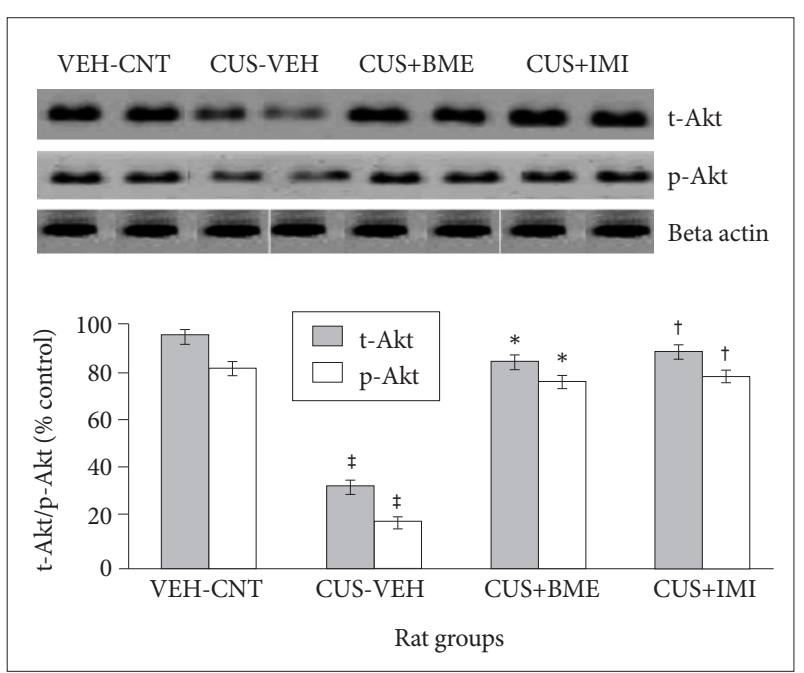

Figure 5. Effect of BME on total Akt and phospho Akt level in the hippocampus in CUS exposed rats. Values given are the mean \pm SEM $(\mathrm{N}=8) .{ }^{*} \mathrm{p}<0.001,{ }^{\dagger} \mathrm{p}<0.001$ as compared with the CUS group, $\neq \mathrm{p}<$ 0.05 as compared with the vehicle control group. VEH: vehicle, CNT: control, CUS: chronic unpredictable stress, BME: Bacopa monnieri extract.

group compared to vehicle control group $(\mathrm{p}<0.001)$. Administration of BME ( $80 \mathrm{mg} / \mathrm{kg}$ b.w.) significantly increased $(\mathrm{p}<0.001)$ total-CREB and phospho-CREB level in hippocampus compared to CUS treated rats, that points out BME can reverse CUS induced de crease of total CREB in hippocampus. Administration of IMI $(20 \mathrm{mg} / \mathrm{kg}$ b.w.) also showed the similar results like BME in hippocampus $(\mathrm{p}<0.001)$.

\section{DISCUSSION}

The present study supports pharmacological and biochemi-

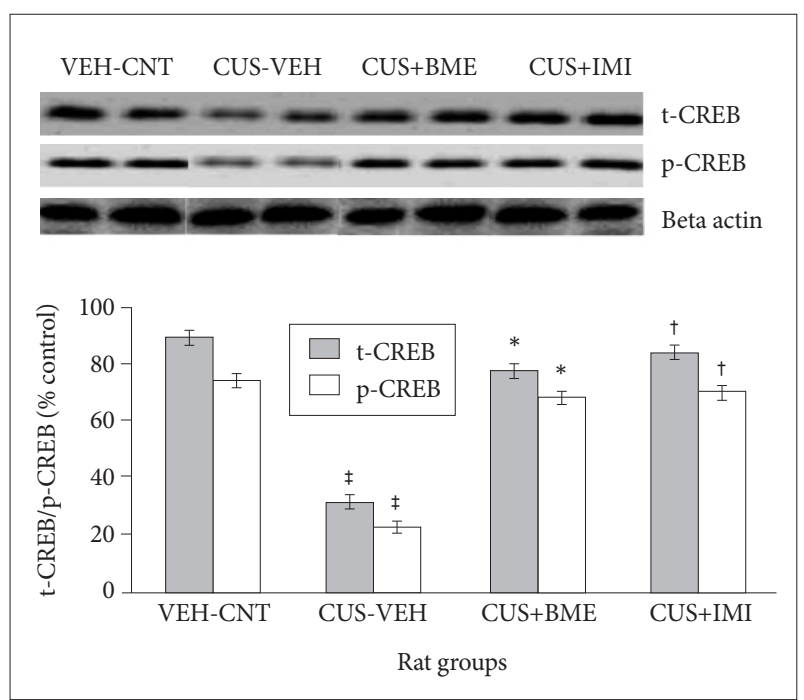

Figure 6. Effect of BME on total CREB and phospho CREB level in the hippocampus in CUS exposed rats. Values given are the mean \pm SEM $(\mathrm{N}=8) .{ }^{*} \mathrm{p}<0.001,{ }^{\dagger} \mathrm{p}<0.001$ as compared with the CUS group, $\neq p<0.05$ as compared with the vehicle control group. VEH: vehicle, CNT: control, CUS: chronic unpredictable stress, BME: Bacopa monnieri extract, IMI: Imipramine.

cal evidences for the antidepressant like effects of BME. Chronic administration of BME $(80 \mathrm{mg} / \mathrm{kg}$ b.w.) reverses all the behavioral changes that occurred due to CUS and restored BDNF, T-Akt, P-Akt, T-CREB and P-CREB protein levels in the hippocampus. IMI, a tricyclic antidepressant was used as a positive control. CUS induced depression has been widely accepted for investigating the pathophysiology of depression. ${ }^{11}$ Several studies pointed out that CUS can induce long term behavioural alterations that are similar with the symptoms of clinical depression..$^{18}$ Hence, CUS causes depression like changes in animal models that can be used to evaluate the efficacy of BME through behavioural tests including the sucrose preference tests, open field tests etc. ${ }^{19,20}$ Anhedonia was assessed by sucrose preference test and rats showed more saccharin solution preference after treatment with BME $\left(80 \mathrm{mg} / \mathrm{kg}\right.$ b.w.). ${ }^{11}$ Behavioral alternations were found in rats after 28 days of CUS exposure in a novel open field test. After CUS exposure, locomotor activities were decreased in CUS grouped rats compared to vehicle controls in the open field test that specifies certain refractory depression. ${ }^{4}$ Chronic administration of BME $(80 \mathrm{mg} / \mathrm{kg}$ b.w. $)$ significantly altered this behavior indicating antidepressant like activity. ${ }^{6}$

Hippocampus is a part of key limbic region of brain that structurally and functionally affected by stress. ${ }^{3}$ Several experiments revealed certain relations between MDD and neuronal atrophy in the hippocampus. ${ }^{21}$ Hippocampus is critically involved in the regulation of mood and memory. ${ }^{3}$ Stress causes reduction in hippocampal volume in depressed patients. ${ }^{22}$ The hippocampus receives signal from the hypothalamic-pituitary- 
adrenal (HPA) axis that modulates stress responses..$^{23}$ It was observed in post-mortem tissues from suicide victims or MDD patients, that BDNF and TrkB levels are decreased in the hippocampus. ${ }^{24} \mathrm{BDNF}$ modulates neuronal plasticity, inhibits cell death cascades and increases the cell survival proteins. ${ }^{25,26}$ Infusion of BDNF into the midbrain, ventricles, or regions of the hippocampus results in increased antidepressant like behaviour. ${ }^{27}$ Several studies provide evidence for the role of BDNF in depression and indicated the up regulation of BDNF expression, may contribute to the action of antidepressants. ${ }^{4}$ In addition, studies in live patients indicate that serum levels of BDNF are normalized in patients suffering from depression after long-term antidepressant treatment ${ }^{14}$ though they have various side effects. ${ }^{4}$ Hence, BDNF can be considered as an important marker in the development and treatment of depression. The present study corroborated with our previous findings and shows that 28 days of CUS exposure significantly decreases BDNF protein levels in the hippocampus while chronic treatment with antidepressant drug inhibits the reuptake of serotonin in the central nervous system. Chronic administration of imipramine hydrochloride or BME show similar results which further indicate that BME can act as an antidepressant. Since we could not measure the active principal in the blood and considered as a limitation of the study.

When BDNF is bound to its cognate receptor TrkB, it induces its dimerization and the receptor tyrosine kinase is autophosphorylated, leading to activation of intracellular signalling cascades. ${ }^{28}$ There are at least three signal transduction pathways that BDNF-TrkB activation can regulate. The phospholipase $\mathrm{C}-\gamma$ (PLC- $\gamma$ ) pathway that leads to activation of protein kinase $\mathrm{C}$, the phosphatidylinositol 3-kinase (PI3K) pathway that activates serine/threonine kinase of Akt, and the mitogenactivated protein kinase (MAPK) or extracellular signal related kinase (ERK) pathway that activates downstream effectors like CREB. ${ }^{16}$ The activation of Akt leads to the phosphorylation of GSK3 $\beta$, which is active in resting cells, but is inactivated by the phosphorylation. The GSK3 $\beta$ has been linked to the regulation of an assembly of transcription factors, including CREB. ${ }^{17}$ In addition, the PI3K/AKT/GSK3 $\beta$ pathways have also emerged as important regulators of depression. The action of BDNF signalling occurs through TrkB-mediated CREB activation, which binds to cAMP response element sites in the BDNF promoter to enhance gene transcription. ${ }^{29}$ Several studies showed recently that the ERK activation is necessary to induce sustained and neuronal activity-dependent CREB phosphorylation. CREB is a critical point of convergence in the signalling pathways regulating neuronal plasticity. ${ }^{30,31}$ The present study is consistent with our recent publication from our laboratory which showed that CUS can cause changes in behavioural pattern among rats. It also showed that due to exposure of CUS, the significant changes have been found in the corticosterone levels, ratio of adrenal gland versus body weight, neurotrophin levels in hippocampus and PFC brain region (BDNF and NGF), Trk B (cognate receptor of BDNF) levels, ERK $1 / 2$ and phospho ERK 1/2 levels. Chronic treatment with BME reverted back those changes significantly ${ }^{3,5,6}$ and suggests the common intracellular pathway for mechanism of antidepressant drug action. ${ }^{32}$ Thus BDNF could protect neurodegeneration through increasing the levels of BDNF, Akt and CREB in the hippocampus.

The present study indicates that chronic administration of BME is able to produce an antidepressant like effect in the CUS induced depression in rats. Furthermore, chronic treatment with BME was effective in reversing the anhedonic and locomotor behaviour in the open field test. These responses may be associated with modulation of signalling pathways linked with BDNF, Akt and CREB. Finally, our results indicate that BME could be an attractive neuro-pharmacological agent for the treatment of neuropsychiatric disorders. The antidepressant effect of BME may be beneficial as a therapy for anxiety and depression. Thus, our results fortify the ethnopharmacological importance of BME in psychiatric disorders like anxiety and MDD. However, more experiment and detailed analysis is required for definitive conclusion.

\section{Acknowledgments}

Special thanks to the Head of the Department of Zoology, University of Calcutta and Raja Peary Mohan College, Uttarpara, Hooghly (W.B.), and School of Life Sciences, JNU for providing infrastructural facilities. We thank our research guide for his timely help, giving outstanding ideas and encouragement to carry on this research work successfully. This work was financially supported by grant from LSRB/ DRDO (Ministry of Defense, Govt. of India) [DLS/81/48222/LSRB-246/EPB/2012] and DST PURSE-II (Govt. of India).

\section{REFERENCES}

1. Shelton RC. The molecular neurobiology of depression. Psychiatr Clin North Am 2007;30:1-11.

2. Kessler RC, Chiu WT, Demler O, Merikangas KR, Walters EE. Prevalence, severity, and comorbidity of 12-month DSM-IV disorders in the National Comorbidity Survey Replication. Arch Gen Psychiatry 2005; 62:617-627.

3. Banerjee R, Hazra S, Ghosh AK, Mondal AC. Chronic administration of bacopa monniera increases BDNF protein and mRNA expressions: a study in chronic unpredictable stress induced animal model of depression. Psychiatry Investig 2014;11:297-306.

4. Mao QQ, Huang Z, Zhong XM, Feng CR, Pan AJ, Li ZY, et al. Effects of SYJN, a Chinese herbal formula, on chronic unpredictable stress-induced changes in behavior and brain BDNF in rats. J Ethnopharmacol 2010;128:336-341.

5. Hazra S, Kumar S, Banerjee R, Banerjee RP, Mondal AC. Bacopa monniera treatment reverses chronic unpredictable stress induced depressive like behavior by increasing expression of neurotrophins in rat brain. RJPBCS 2013;4:775-786.

6. Hazra S, Kumar S, Saha GK, Mondal AC. Chronic administration of bacopa monniera alleviates depressive like behavior and increases the 
expression of ERK1/2 in hippocampus and pre frontal cortex of chronic unpredictable stress induced rats. Int Neuropsychiat Dis J 1 2015;3:4758.

7. Zhang ZJ. Therapeutic effects of herbal extracts and constituents in animal models of psychiatric disorders. Life Sci 2004;75:1659-1699.

8. Calabrese C, Gregory WL, Leo M, Kraemer D, Bone K, Oken B. Effects of a standardized Bacopa monnieri extract on cognitive performance, anxiety, and depression in the elderly: a randomized, doubleblind, placebo-controlled trial. J Altern Complement Med 2008;14:707713.

9. Paulose CS, Chathu F, Khan SR, Krishnakumar A. Neuroprotective role of Bacopa monnieri extract in epilepsy and effect of glucose supplementation during hypoxia: glutamate receptor gene expression. Neurochem Res 2008;33:1663-1671.

10. Chatterjee M, Verma P, Palit G. Comparative evaluation of Bacopa monniera and Panax quniquefolium in experimental anxiety and depressive models in mice. Ind J Exp Biol 2010;48:306-313.

11. Willner P. Chronic mild stress (CMS) revisited: consistency and behavioural neurobiological concordance in the effects of CMS. Neuropsychobiology 2005;52:90-110.

12. Banerjee R, Hazra S, Kumar S, Ghosh AK, Mondal AC. Behavioral consequences of chronic stress and effects of antidepressant treatment on animal models of depression. Am J Psychiatry Neurosci 2013;1:5-13.

13. Kossel AH, Cambridge SB, Wagner U, Bonhoeffer T. A caged Ab reveals an immediate/instructive effect of BDNF during hippocampal synaptic potentiation. Proc Natl Acad Sci U S A 2001;98:14702-14707.

14. Duman RS, Monteggia LM. A neurotrophic model for stress-related mood disorders. Biol Psychiatry 2006;59:1116-1127.

15. Yang J, Siao CJ, Nagappan G, Marinic T, Jing D, McGrath K, et al. Neuronal release of pro BDNF. Nat Neurosci 2009;12:113-115.

16. Mattson MP. Glutamate and neurotrophic factors in neuronal plasticity and disease. Ann N Y Acad Sci 2008;1144:97-112.

17. Yoshii A, Constantine-Paton M. Postsynaptic BDNF-TrkB signaling in synapse maturation, plasticity, and disease. Dev Neurobiol 2010;70:304322.

18. Luo DD, An SC, Zhang X. Involvement of hippocampal serotonin and neuropeptide $\mathrm{Y}$ in depression induced by chronic unpredicted mild stress. Brain Res Bull 2008;77:8-12.

19. Li S, Wang C, Wang M, Li W, Matsumoto K, Tang Y. Antidepressant like effects of piperine in chronic mild stress treated mice and its possible mechanisms. Life Sci 2007;80:1373-1381.

20. Zhao Z, Wang W, Guo H, Zhou D. Antidepressant-like effect of liquiri- tin from Glycyrrhiza uralensis in chronic variable stress induced depression model rats. Behav Brain Res 2008;194:108-113.

21. Fuchs E, Czeh B, Kole MH, Michaelis T, Lucassen PJ. Alterations of neuroplasticity in depression: the hippocampus and beyond. Eur Neuropsychopharmacol 2004;14(Suppl 5):S481-S490.

22. Bremner JD, Narayan M, Anderson ER, Staib LH, Miller HL, Charney DS. Hippocampal volume reduction in major depression. Am J Psychiatry 2000;157:115-118.

23. McEwen BS, Magarinos AM. Stress effects on morphology and function of the hippocampus. Ann NY Acad Sci 1997;821:271-284.

24. Banerjee R, Ghosh AK, Ghosh B, Bhattacharyya S, Mondal AC. Decreased mRNA and protein expressions of BDNF, NGF and their receptors in the hippocampus from suicide: an analysis in human postmortem brain. Clin Med Insights Pathol 2013;6:1-11.

25. Castrén E, Võikar V, Rantamäki T. Role of neurotrophic factors in depression. Curr Opin Pharmacol 2007;7:18-21.

26. Castrén E, Rantamäki T. The role of BDNF and its receptors in depression and antidepressant drug action: reactivation of developmental plasticity. Dev Neurobiol 2010;70:289-297.

27. Shirayama Y, Chen AC, Nakagawa S, Russell DS, Duman RS. Brain derived neurotrophic factor produces antidepressant effects in behavioral models of depression. J Neurosci 2002;22:3251-3261.

28. Levine ES, Crozier RA, Black IB, Plummer MR. Brain-derived neurotrophic factor modulates hippocampal synaptic transmission by increasing N-methyl-D-aspartic acid receptor activity. Proc Natl Acad Sci U S A 1998;95:10235-10239.

29. Lu Y, Christian K, Lu B. BDNF: a key regulator for protein synthesis dependent LTP and long-term memory? Neurobiol Learn Mem 2008; 89:312-323.

30. Qi XL, Lin WJ, Li JF, Pan YQ, Wang WW, Sun M. A role for the extracellular signal-regulated kinase signal pathway in depressive-like behavior. Behav Brain Res 2009;199:203-209.

31. Qi XL, Lin WJ, Li JF, Li HH, Wang WW, Wang DL, et al. Fluoxetine increases the activity of the ERK-CREB signal system and alleviates the depressive like behavior in rats exposed to chronic forced swim stress. Neurobiol Dis 2008;31:278-285.

32. Rantamäki T, Hendolin P, Kankaanpää A, Mijatovic J, Piepponen P, Domenici E, et al. Pharmacologically diverse antidepressants rapidly activate brain-derived neurotrophic factor receptor TrkB and induce phospholipase-C gamma signaling pathways in mouse brain. Neuropsychopharmacology 2007;32:2152-2162. 